Revista Mexicana de Astronomía y Astrofísica, 57, 407-418 (2021)

(c) 2021: Instituto de Astronomía, Universidad Nacional Autónoma de México

https://doi.org/10.22201/ia.01851101p.2021.57.02.13

\title{
STABILITY ANALYSIS OF RELATIVISTIC POLYTROPES
}

\author{
Abdel-Naby S. Saad ${ }^{1,2}$, Mohamed I. Nouh ${ }^{2}$, Ashraf A. Shaker ${ }^{2}$, and Tarek M. Kamel ${ }^{2}$
}

Received March 29 2021; accepted July 232021

\begin{abstract}
We study the relativistic self-gravitating, hydrostatic spheres with a polytropic equation of state, considering structures with the polytropic indices $n=1(0.5) 3$ and illustrate the results for the relativistic parameters $\sigma=0-0.75$. We determine the critical relativistic parameter at which the mass of the polytrope has a maximum value and represents the first mode of radial instability. For $n=1(0.5) 2.5$, stable relativistic polytropes occur for $\sigma$ less than the critical values $0.42,0.20,0.10$, and 0.04 , respectively, while unstable relativistic polytropes are obtained when $\sigma$ is greater than the same values. When $n=3.0$ and $\sigma>0.5$, energetically unstable solutions occur. The results of critical values are in full agreement with those evaluated by several authors. Comparisons between analytical and numerical solutions of the given relativistic functions provide a maximum relative error of order $10^{-3}$.
\end{abstract}

\section{RESUMEN}

Estudiamos esferas hidrostáticas, autogravitantes y relativistas, con una ecuación de estado politrópica, considerando estructuras con índices politrópicos $n=1(0.5) 3$, e ilustramos los resultados para parámetros relativistas $\sigma=0-0.75$. Determinamos el parámetro relativista crítico, para el cual la masa del politropo alcanza un valor máximo y representa el primer modo de inestabilidad radial. Con $n=1(0.5) 2.5$ encontramos politropos relativistas estables para $\sigma$ menor que los valores críticos $0.42,0.20,0.10$, y 0.04 , respectivamente. Se obtienen politropos relativistas inestables para valores mayores de $\sigma$. Cuando $n=3.0$ y $\sigma>0.5$ encontramos soluciones energéticamente inestables. Los resutados sobre los valores críticos concuerdan muy bien con los de otros autores. Al comparar las soluciones analíticas y numéricas de las funciones relativistas estudiadas se encuentran errores relativos máximos del orden de $10^{-3}$.

Key Words: methods: analytical — methods: numerical — stars: interiors

\section{INTRODUCTION}

Polytropic models could be considered simple models of stellar structure, and we have all of the equations needed to make more sophisticated stellar models by solving the equations of stellar structure. However, there is a need to ask whether the calculated models provide stable spherically symmetric models.

Several authors have investigated the stability of the polytropic models. Bonnor (1958) found that self-gravitating, polytropic spheres with $n=3$ were inconditionally stable to radial perturbations. For

\footnotetext{
${ }^{1}$ Department of Mathematics, Deanship of Educational Services, Qassim University, Buraidah, Saudi Arabia.

${ }^{2}$ Astronomy Department, National Research Institute of Astronomy and Geophysics(NRIAG), 11421 Helwan, Cairo, Egypt.
}

the first time, Chandrasekhar (1964) provided the radial stability equation. Earlier methods used to examine the stability of polytropic stars are listed in Bardeen et al. (1966). More recently, the stability of polytropes with different polytropic indices was described by Horedt (2013) and Raga et al. (2020).

In stellar structures such as white dwarfs, neutron stars, black holes and supermassive stars, and in star clusters, relativistic effects play a significant role (Sen and Roy 1954, Sharma 1988). Tooper (1964) performed a relativistic analysis of the polytropic equation of state and derived the non-relativistic Lane-Emden equation from two nonlinear differential equations (Tolman-Oppenheimer, TOV). The problem of the stability of relativistic stars has long been investigated in the literature, for example, by 
Zeldovich and Novikov (1978), Shapiro and Teukolsky (1984), Takatsuka and Tamagaki (1993), Casalbuoni and Nardulli (2004), Khalilov (2002), Isayev (2015), Chu et al. (2015).

In the present paper, we examine the stability of the relativistic polytrope for different polytropic indices. An analytical solution to the TOV equation is introduced, which provides the physical parameters of the relativistic polytrope. We investigate the critical values of the relativistic parameter for which the onset of the radial instability occurs. The structure of the paper is as follows: $\S 2$ is devoted to the formulation of the TOV equation. In $\S 3$ we give a brief description of the analytical method used to solve the TOV equation. $\S 4$ deals with the obtained results. The conclusion is outlined in $\S 5$.

\section{THE EQUATION OF HYDROSTATIC EQUILIBRIUM}

The interior of a symmetric star can be described in a spherical coordinate system $(r, \vartheta, \varphi)$ by the standard form of the metric (Tolman 1939, Landu \& Lifshitz 1975)

$$
d s^{2}=e^{\nu} c^{2} d t^{2}-e^{\lambda} d r^{2}-r^{2} d \vartheta^{2}-r^{2} \sin ^{2} \vartheta d \varphi^{2},
$$

where $\nu$ and $\lambda$ are functions of radius $r$. As for a fluid star, the components of the energy momentum tensor corresponding to the above metric are given by

$$
T_{0}^{0}=\rho c^{2} e^{\nu}, \quad T_{1}^{1}=P e^{\lambda}, T_{2}^{2}=P r^{2}, T_{3}^{3}=P r^{2} \sin ^{2} \vartheta,
$$

where $\rho, P$ and $c$ are the mass density, pressure, and speed of light, respectively. The time-independent gravitational equations for the line element equation (1) and the energy momentum tensor are

$$
\begin{gathered}
e^{-\lambda}\left(\frac{1}{r} \frac{d \nu}{d r}+\frac{1}{r^{2}}\right)-\frac{1}{r^{2}}=\frac{8 \pi G}{c^{4}} P, \\
e^{-\lambda}\left(\frac{1}{r} \frac{d \lambda}{d r}-\frac{1}{r^{2}}\right)+\frac{1}{r^{2}}=\frac{8 \pi G}{c^{4}} \rho c^{2}, \\
\frac{d P}{d r}=-\frac{1}{2}\left(P+\rho c^{2}\right) \frac{d \nu}{d r},
\end{gathered}
$$

where $G=6.67 \times 10^{-8} \mathrm{~g}^{-1} \mathrm{~cm}^{3} \mathrm{~s}^{-2}$ is the Newtonian gravitational constant. Equations (3), (4), and (5) together with the equation of state $\rho=\rho(P)$ represent the hydrostatic equilibrium for an isotropic general relativistic fluid sphere and can be solved to get $\lambda, \nu, P$ and $\rho$ as functions of $r$. For hydrostatic equilibrium stars, the Tolman-Oppenheimer-Volkoff
(TOV) general relativity equation obtained by solving Einstein's field equations has the form

$$
\begin{aligned}
\frac{d P}{d r}= & -\frac{G \varepsilon(r) m(r)}{c^{2} r^{2}}\left[1+\frac{P(r)}{\varepsilon(r)}\right]\left[1+\frac{4 \pi r^{3} P(r)}{m(r) c^{2}}\right] \\
& {\left[1-\frac{2 G m(r)}{c^{2} r}\right]^{-1}, }
\end{aligned}
$$

where

$$
m(r)=\int_{0}^{r} 4 \pi \rho(r) r^{2} d r,
$$

is the gravitational mass interior to radius $r$ and $\varepsilon(r)$ is the internal energy density.

Equation (6) is an extension of the Newtonian formalism with a relativistic correction. The equation of state for a polytropic star is $P=K \rho^{1+\frac{1}{n}}$, where $n$ is the polytropic index. Tooper (1964) has shown that the TOV equation together with the mass conservation equation have the form

$$
\xi^{2} \frac{d \theta}{d \xi} \frac{1-2 \sigma(n+1) v / \xi}{1+\sigma \theta}+v+\sigma \xi \theta \frac{d v}{d \xi}=0,
$$

and

$$
\frac{d v}{d \xi}=\xi^{2} \theta^{n}
$$

with the initial conditions

$$
\theta(0)=1, \quad v(0)=0,
$$

where

$$
\begin{aligned}
& \theta=\rho / \rho_{c}, \quad \xi=r A, \quad v=\frac{A^{3} m(r)}{4 \pi \rho_{c}}, \\
& A=\left(\frac{4 \pi G \rho_{c}}{\sigma(n+1) c^{2}}\right)^{1 / 2}, \sigma=\frac{P_{c}}{\rho_{c} c^{2}}=\frac{K \rho_{c}{ }^{1 / n}}{c^{2}},
\end{aligned}
$$

$\sigma$ is the relativistic parameter that can be related to the sound velocity in the fluid, because the sound velocity is given by $v_{s}^{2}=\frac{d P}{d \rho}$ in an adiabatic expression. In equations (10) $\theta, \xi$ and $v$ are dimensionless parameters, while $A$ is a constant.

If the pressure is much smaller than the energy density at the center of a star (i.e. $\sigma$ tends to zero), then equation (7) reduces to

$$
\xi^{2} \frac{d \theta}{d \xi}+v=0 .
$$

Equation (8) together with equation (11) reproduce the well-known Lane-Emden equation for Newtonian polytropic stars

$$
\frac{1}{\xi^{2}} \frac{d}{d \xi}\left(\xi^{2} \frac{d \theta}{d \xi}\right)+\theta^{n}=0 .
$$


When $n$ tends to zero, we obtain the case of incompressible matter, for which the analytic solutions are possible in both relativistic and nonrelativistic cases. The nonrelativistic Lane-Emden equation has an analytical solution in closed form only for $n=0,1$ and 5. However, this is not possible for the relativistic equation, and numerical integrations must be performed (Tooper 1964; Bludman 1973; Ferrari et al. 2007).

\section{ANALYTICAL SOLUTIONS OF THE RELATIVISTIC EQUATIONS}

Nouh (2004), Nouh and Saad (2013) introduced a new analytical solution of equations (7-8) applying the Euler-Abel transformation (Demodovich \& Maron, 1973) and then a Pade approximation to the Euler-Abel transformed series (Appendix B) to accelerate the convergence of the power series solutions.

In this paper, we analyze the gravitational stability of polytropic fluid spheres based on the analytical solution of the TOV equations that have already been given by Nouh and Saad (2013). We consider the cases of polytropic index $n=3.0,2.5,2.0,1.5$, and 1.0 for $\sigma<n /(n+1)$.

The analytical solution has the form:

$$
\theta(\xi)=1+\sum_{k=1}^{\infty} a_{k} \xi^{2 k},
$$

where

$$
\begin{aligned}
a_{k+1}= & \frac{\sigma}{2(k+1)}\left(2(n+1) \gamma_{k-1}-\eta_{k}-\beta_{k}+\sigma \zeta_{k}\right)- \\
& \frac{\alpha_{k}}{2(k+1)(2 k+3)}, \quad k \geq 1, \\
\gamma_{k-1}= & \sum_{i=0}^{k-1} f_{i} g_{k-i-1}, \quad \eta_{k}=\sum_{i=0}^{k} a_{i} g_{k-i}, \\
\beta_{k}= & \sum_{i=0}^{k} a_{i} \alpha_{k-i}, \quad \zeta_{k}=\sum_{i=0}^{k} a_{i} \beta_{k-i}, \\
f_{i}= & 2(i+1) a_{i+1}, g_{i}=\frac{\alpha_{i}}{(2 i+3)}, \quad \gamma_{k}=\sum_{i=0}^{k} f_{i} g_{k-i}, \\
\alpha_{k}= & \frac{1}{k a_{0}} \sum_{i=1}^{k}(n i-k+i) a_{i} \alpha_{k-i}, \quad k \geq 1, \\
\alpha_{0}= & a_{0}^{n}, \text { and } a_{0}=1 .
\end{aligned}
$$

From equations (10), for some values of $n, \sigma$ and $\rho_{c}$ we can determine $K$, and obtain the radius $R$ and the mass $M(R)$ from

$$
\begin{gathered}
R=A^{-1} \xi_{1}=\left[\frac{c^{2}}{4 \pi G}(n+1) \sigma^{(1-n)}\left(\frac{K}{c^{2}}\right)^{n}\right]^{1 / 2} \xi_{1}, \\
M=\frac{4 \pi \rho_{c}}{A^{3}} \nu\left(\xi_{1}\right)=\left[\frac{1}{4 \pi}\left(\frac{(n+1) c^{2}}{G}\right)^{3}\left(\frac{K}{c^{2}}\right)^{n}\right]^{1 / 2} \tilde{M}, \\
\tilde{M} \equiv \sigma^{(3-n) / 2} \nu\left(\xi_{1}\right) .
\end{gathered}
$$

$\xi_{1}$ is the first zero of the Lane-Emden function $\theta(\xi)$; equation (8), can be written in the form

$$
v\left(\xi_{1}\right)=\sum_{k=0}^{\infty} \frac{\alpha_{k}}{(2 k+3)} \xi_{1}{ }^{2 k+3} .
$$

The power series solution, equation (13), converges rapidly for values of the polytropic index $n \leq 2$ and the error between analytical and numerical solutions is of order $10^{-4}$. For $n>2$, the series solution utilized gives a slow convergence, and the calculation of the stellar mass from equations (16) and (17) indicates a larger error. The physical range for a convergent power series can be extended with a change of the independent variable. Transformations by changing the independent variable are utilized to improve and accelerate the series convergence in equation (18) for $n>2$ (Pasucal 1977, Saad 2004):

$$
x=6 *\left\{\left(1+\frac{1}{3} \xi^{2}\right)\right\}^{1 / 2} .
$$

\section{RESULTS}

The results evaluated by the use of equations in $\S 3$ are utilized here to analyze the stability of relativistic polytropes for various values of the general relativity parameter $\sigma$ and polytropic index $n$. The numerical results obtained are tabulated in Appendix A for $n=$ $1(0.5)$ to 3.0 and a range of values of $\sigma$. Comparisons of the analytical solutions of equation (1) and $\tilde{M}(\sigma)$ to the numerical method are given in Tables 5 to 9 . Table 10 shows the critical values of $\tilde{M}(\sigma)$ due to relativistic effects for different polytropic indices.

In Figures 1 to 5 we plot $\tilde{M}$, equation (17), as a function of the index $n$ and the relativistic effect $\sigma$. The figures show an increase of $\tilde{M}$ (consequently an increase of the stellar mass $M$ ) with $\sigma$ up to some maximum values (say, $\sigma_{C R}$ ). It is worth mentioning that the critical value $\sigma_{C R}$ marks 


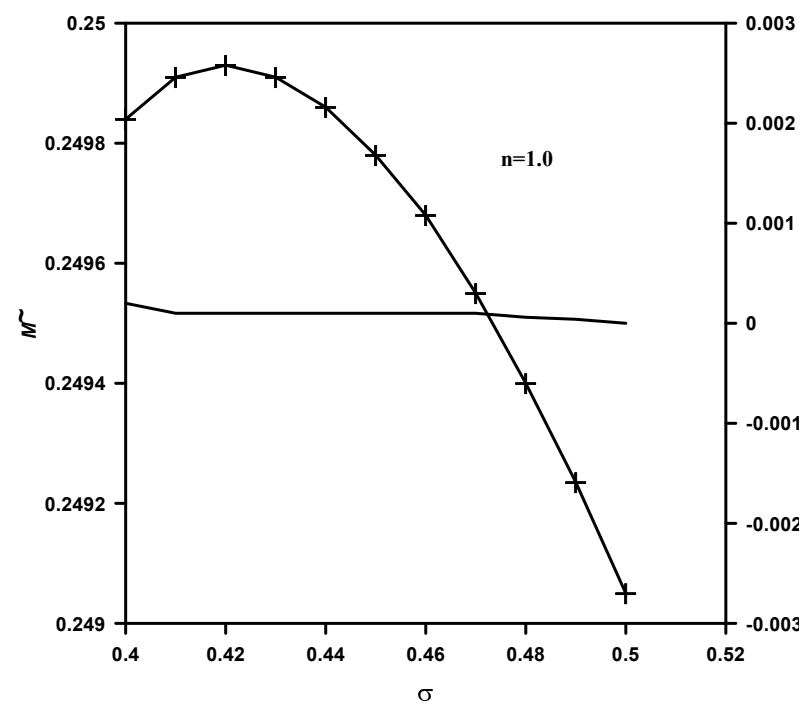

Fig. 1. $\tilde{M}(\sigma)$ for $n=1$, and $\sigma_{C R} \approx 0.42$. Stable relativistic polytropes occur for $\sigma<0.42$, while unstable models occur when $\sigma>0.42$. Comparison of analytical and numerical results provides errors of order $10^{-4}$.

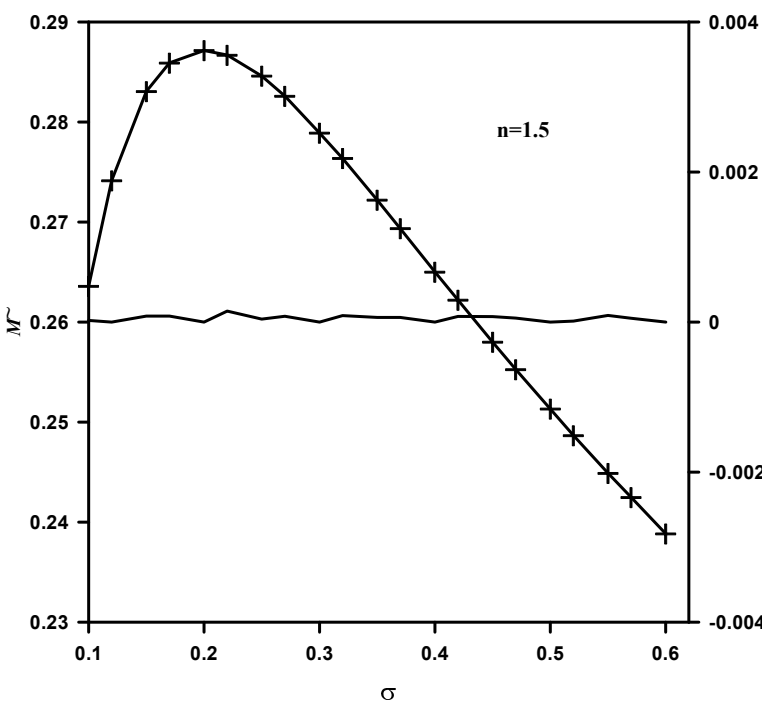

Fig. 2. $\tilde{M}(\sigma)$ for $n=1.5$, and $\sigma_{C R} \approx 0.2$. Stable relativistic polytropes occur for $\sigma<0.2$, while unstable models occur when $\sigma>0.2$. Comparison of analytical and numerical results provides errors of order $10^{-5}$.

the onset of the first mode of radial instability. For the case $n=1.0$, Figure 1 shows that the critical value $\sigma_{C R}=0.42$, and the relativistic polytropes are stable for $\sigma<0.42$. In Figures 2, 3, and 4 we observe critical values of the general relativity index $\sigma_{C R}=0.2, \sigma_{C R}=0.1$ and $\sigma_{C R}=0.04$ for the cases

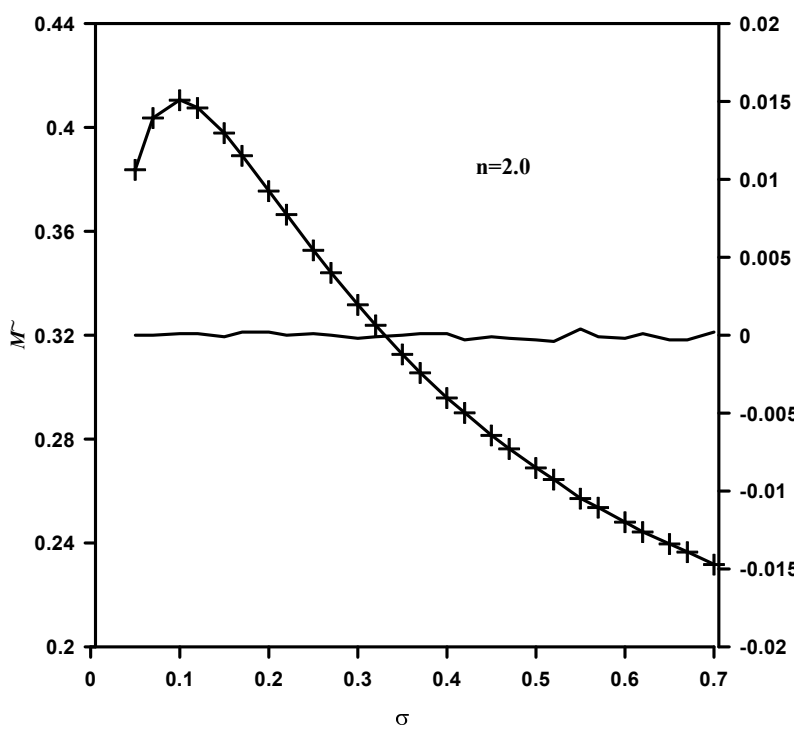

Fig. 3. $\tilde{M}(\sigma)$ for $n=2$, and $\sigma_{C R} \approx 0.1$. Stable relativistic polytropes occur for $\sigma<0.1$, while unstable models occur when $\sigma>0.1$. Comparison of analytical and numerical results provides errors of order $10^{-4}$.

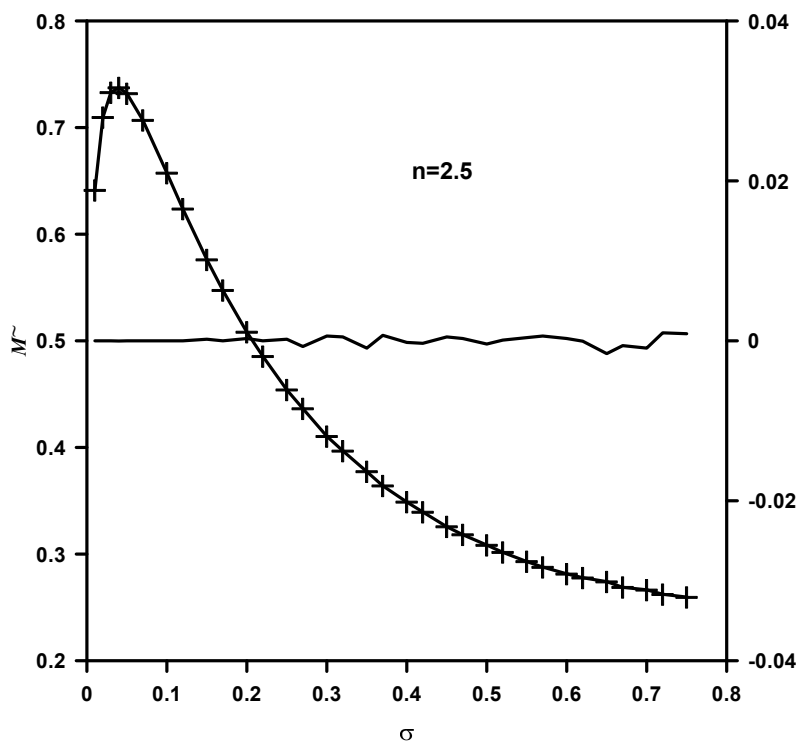

Fig. 4. $\tilde{M}(\sigma)$ for $n=2.5$, and $\sigma_{C R} \approx 0.04$. Stable relativistic polytropes occur for $\sigma<0.04$, while unstable models occur when $\sigma>0.04$. Comparison of analytical and numerical results provides errors of order $10^{-4}$.

$n=1.5, n=2.0$ and $n=2.5$ respectively. In Figure 5 where $n=3.0, \tilde{M}$ has a maximum at $\sigma_{C R}=0$ which marks the onset of the first mode of instability, while the minimum value at $\sigma_{C R}=0.53$ marks the onset of the next mode of nonradial instability. In this case, equation (17) reduces to $\tilde{M} \equiv \nu\left(\xi_{1}\right)$. We 


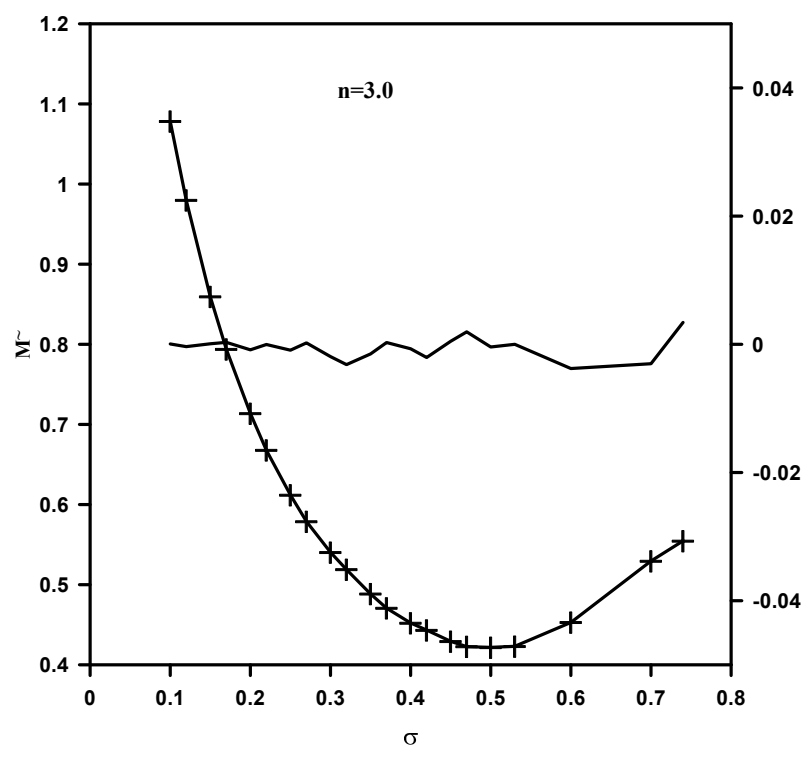

Fig. 5. For $n=3.0, \tilde{M}$ has a maximum at $\sigma_{C R}=0$ which marks the onset of the first mode of instability, while the minimum at $\sigma_{C R} \approx 0.53$ marks the onset of the next mode of nonradial instability. Comparison of analytical and numerical results provides errors of order $10^{-3}$.

conclude that for $\sigma_{C R}>0.5$ the relativistic polytropic models are energetically unstable.

The study of the stability of polytropes is useful for determining some physical properties, such as the maximum mass limit, and illustrates how the stellar mass increases or decreases due to the effects of general relativity. For a given mass, radius, and a polytropic index $n$, Figure 6 of the massradius relation can be used to determine the internal structure of a polytrope. This means that each value of a relativistic parameter $\sigma$ corresponds to a certain internal structure. We can see from Figure 6 that one pair of mass and radius corresponds to two different values of $\sigma$. For the case of a polytropic index $n=3.0$, the logarithmic function $\left.\log _{10}\left[\sigma(n+1) v\left(\xi_{1}\right) / \xi_{1}\right)\right]=-2.03$ has two values of $\sigma \simeq 0.67$ and $\sigma \simeq 0.75$. Then we have two spherical polytropic configurations of the same mass and radius, but with different internal structures. When $n=2.0$, the logarithmic function $\left.\log _{10}\left[\sigma(n+1) v\left(\xi_{1}\right) / \xi_{1}\right)\right]=-0.76$ has two values of $\sigma \simeq 0.42$ and $\sigma \simeq 0.47$. Such information reflects the importance of relativistic solutions.

Table 1 gives the limits of the mass-radius relations; for example, if the polytropic index $n=1.0 G M / c^{2} \bar{R} \leq 0.214$, then the gravitational radius $2 G M / c^{2}$ is at most $43 \%$ of the invariant(physical) radius $\bar{R}$. When the polytropic index

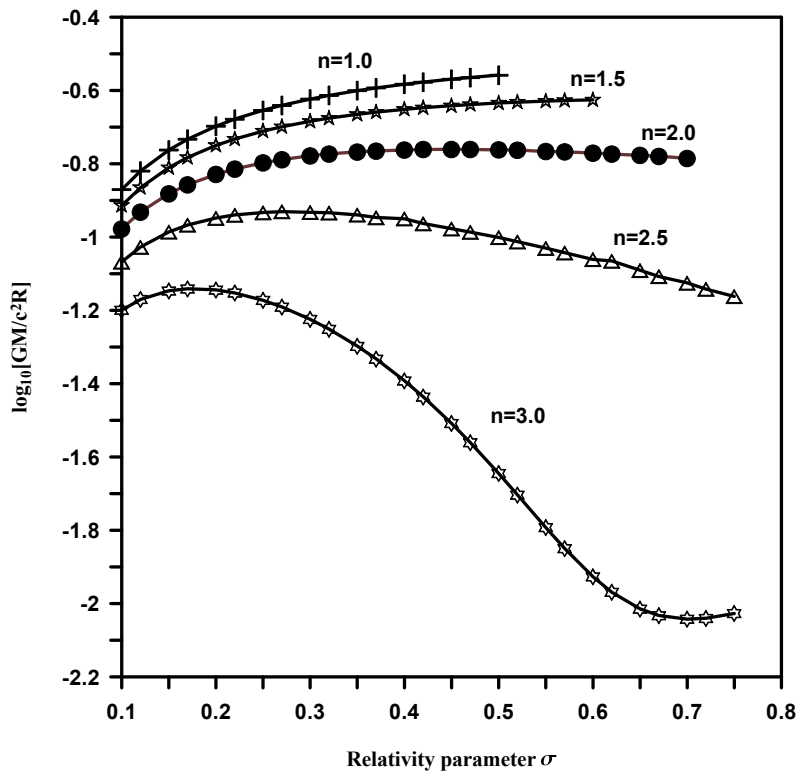

Fig. 6. The logarithmic ratio of the gravitational radius to the coordinate radius, as a function of the relativistic parameter $\sigma$, for different values of the polytropic index $n$.

$n=3.0 \mathrm{GM} / \mathrm{c}^{2} \bar{R} \leq 0.072$, then the gravitational radius $2 \mathrm{GM} / \mathrm{c}^{2}$ is at most $14.5 \%$ of the invariant radius $\bar{R}$, which is very small compared to the limit value when $n=1.0$.

The results of all critical values obtained in this paper for different polytropic indices are in full agreement with those evaluated by several authors such as Tooper(1964), Bludman(1973), and Araujo \& Chirenti (2011). These critical values $\sigma_{C R}$ and $\tilde{M}(\sigma)$ together with various indices $n$ are given in Table 10 (Appendix A). It is shown that the spherical polytrope of index $n=3.0$ and $\sigma>0.5$ is energetically unstable.

The mass-radius relation (Tooper 1964) has the form:

$$
\frac{G M}{c^{2} \bar{R}}=\frac{\sigma(n+1) v\left(\xi_{1}\right)}{\overline{\xi_{1}}},
$$

where $\bar{R}$ defines the physical radius (invariant radius) of the sphere and $\overline{\xi_{1}}=A \bar{R}$ can be obtained by integrating the equation

$$
\overline{\xi_{1}}=\int_{0}^{\xi_{1}}(1-2 \sigma(n+1) v(\xi) / \xi)^{-1 / 2} d \xi .
$$

The mass-radius relation is useful for determining the surface redshift. It gives the ratio of the gravitational radius $2 G M / c^{2}$ to the invariant radius $\bar{R}$ when $n$ and $\sigma$ are known. Rewrite equation (20) in 
TABLE 1

LIMITS OF THE MASS-RADIUS RELATIONS

\begin{tabular}{cccc}
\hline$n$ & Max. value of $\log _{10}\left[\sigma(n+1) v\left(\xi_{1}\right) / \overline{\xi_{1}}\right]$ & Limit ratio of $G M / c^{2} \bar{R}$ & Limit ratio of $G M / c^{2} R$ \\
\hline 1.0 & -0.670 & 0.214 & 0.277 \\
1.5 & -0.769 & 0.170 & 0.237 \\
2.0 & -0.885 & 0.130 & 0.174 \\
2.5 & -1.022 & 0.095 & 0.117 \\
3.0 & -1.201 & 0.0633 & 0.072 \\
\hline
\end{tabular}

terms of numerical values for solar mass and solar radius and take logarithms of both sides of the resulting equation (Tooper, 1964). Then using the solutions introduced in $\S 3$, we plotted the logarithmic ratio of gravitational radius to a geometrical radius as a function of the relativistic parameter for different values of the polytropic index $n$ (see Figure 6). Table 1 gives the limits of the mass-radius relations for different polytropic index $n$.

\section{SERIES CONVERGENCE}

The power series solution of the relativistic problem without using any acceleration techniques is very limited. Tables 2,3 , and 4 show the radius of convergence $\xi_{1}$ of the power series solution (1) of equation (13) and the relative error $(\varepsilon)$ before performing any acceleration. For the polytropic indices $n=1.0$ and $n=1.5$, the series is rapidly convergent. However, beyond these values, the power series solution is either slowly convergent or divergent. Note that the relative error $\left(\varepsilon=\left|\xi_{1}(A n)-\xi_{1}(N u m)\right| / \xi_{1}(N u m)\right)$ increases gradually with relativistic effect $\sigma$ and polytropic index $n$. This in turn results in a small physical range for the convergent power series solutions, and may produce inaccurate physical parameters of the relativistic polytropes.

The fourth order Runge-Kutta method was used for the performance of the numerical solution of the relativistic TOV equation. Analytical and numerical calculations were done using the Mathematica package, version 11.2.

To extend the physical radii of the convergent power series solutions, a combination of the two techniques for Euler-Abel transformation and Padé approximation (Nouh 2004; Nouh \& Saad 2013) were utilized. Tables 5 to 9 (Appendix A) show comparisons between numerical and analytical results. It is worth noting that the power series solutions are rapidly convergent for polytropic indices $n=1(0.5) 3.0$ and provide a maximum relative error of order $10^{-3}$.
TABLE 2

RADII OF CONVERGENCE OF $\theta(\xi)$ AND RELATIVE ERROR FOR $n=1.0$

\begin{tabular}{cccc}
\hline$\sigma$ & $\xi_{1}(N)$ & $\xi_{1}(A)$ & $\varepsilon:$ relative error \\
\hline 0.1 & 2.5990 & 2.5990 & 0.0 \\
0.2 & 2.2770 & 2.2765 & 0.000219635 \\
0.3 & 2.0641 & 2.0637 & 0.000193827 \\
0.4 & 1.9132 & 1.9111 & 0.001098844 \\
0.5 & 1.8008 & 1.8862 & 0.045276217 \\
\hline
\end{tabular}

TABLE 3

RADII OF CONVERGENCE OF $\theta(\xi)$ AND RELATIVE ERROR FOR $n=1.5$

\begin{tabular}{cccc}
\hline$\sigma$ & $\xi_{1}(N)$ & $\xi_{1}(A)$ & $\varepsilon:$ relative error \\
\hline 0.1 & 3.0384 & 3.0730 & 0.011259356 \\
0.2 & 2.6993 & 2.6025 & 0.037195005 \\
0.3 & 2.4930 & 2.4281 & 0.026728718 \\
0.4 & 2.3610 & 2.2648 & 0.042476157 \\
0.5 & 2.2749 & 2.0644 & 0.101966673 \\
0.6 & 2.2192 & 1.8340 & 0.210032715 \\
\hline
\end{tabular}

TABLE 4

RADII OF CONVERGENCE OF $\theta(\xi)$ AND RELATIVE ERROR FOR $n=2.0$

\begin{tabular}{cccc}
\hline$\sigma$ & $\xi_{1}(N)$ & $\xi_{1}(A)$ & $\varepsilon$ : relative error \\
\hline 0.1 & 3.6989 & 3.4259 & 0.07968709 \\
0.2 & 3.3983 & 2.5632 & 0.325803683 \\
0.3 & 3.2711 & 2.5577 & 0.278922469 \\
0.4 & 3.2473 & 1.9503 & 0.665025893 \\
0.5 & 3.2967 & 1.9836 & 0.661978221 \\
0.6 & 3.3986 & 1.7686 & 0.92163293 \\
0.67 & 3.4982 & 1.6556 & 1.112949988 \\
\hline
\end{tabular}




\section{CONCLUSION}

In the present paper, we study the stability properties of the relativistic polytrope. We analyze for various polytropic indices the stability of the relativistic polytrope. An analytic solution is applied to the TOV equation that provides us with relativistic polytropic physical parameters. For each polytropic index, we test the critical values of the relativistic parameter at which the radial instability started. It is shown that for a given mass, radius and polytropic index $n$, the internal structure of a polytropic fluid sphere can be determined as a function of the relativistic parameter $\sigma$. For $n=1(0.5) 2.5$, stable relativistic polytropes occur for $\sigma$ less than the critical values $0.42,0.20,0.10$, and 0.04 , respectively, while unstable relativistic polytropes are obtained when the relativistic parameter $\sigma$ is greater than the same values. When $n=3.0$ and $\sigma>0.5$, energetically unstable solutions occur.

We thank the referee for his/her valuable comments which improved the paper.

\section{APPENDIX A: NUMERICAL RESULTS}

In the following tables, we list the numerical results obtained for different polytropic indices. The designation of the columns are as follows:

1. $\sigma$ : is the relativistic parameter.

2. $\xi_{1}$ : is the first zero of the Emden function.

3. $\nu\left(\xi_{1}\right)_{N u m}$ : is the numerical solution of the relativistic function.

4. $\nu\left(\xi_{1}\right)_{A n}$ : is the analytical solution of the relativistic function.

5. $\tilde{M}(\sigma)_{N u m}$ : is a parameter analog to the mass of the polytrope computed numerically.

6. $\tilde{M}(\sigma)_{A n}$ : is a parameter analog to the mass of the polytrope computed analytically.

7. $\Delta \nu\left(\xi_{1}\right)_{N u m}$ : is the difference between the analytical and the numerical values of the function.

8. $\Delta \tilde{M}(\sigma)_{A n}$ : is the difference between the analytical and the numerical values.

9. $\sigma_{\text {critical }}$ : is the critical value of the fractional parameter at which instability started.

TABLE 5

COMPARISONS BETWEEN ANALYTICAL AND NUMERICAL SOLUTIONS OF THE RELATIVISTIC FUNCTIONS (1) AND $\tilde{M}(\sigma)$ FOR $n=1.0$

\begin{tabular}{|c|c|c|c|c|c|c|c|}
\hline$\sigma$ & $\xi_{1}$ & $\nu\left(\xi_{1}\right)_{N u m}$ & $\nu\left(\xi_{1}\right)_{A n}$ & $\tilde{M}(\sigma)_{N u m}$ & $\tilde{M}(\sigma)_{A n}$ & $\Delta \nu\left(\xi_{1}\right)_{N u m}$ & $\Delta \tilde{M}(\sigma)_{A n}$ \\
\hline 0.0 & 3.1415 & 3.1416 & 3.1416 & 0.0 & 0.0 & 0.0 & 0.0 \\
\hline 0.10 & 2.5990 & 1.7514 & 1.7514 & 0.1751 & 0.1751 & 0.0 & 0.0 \\
\hline 0.12 & 2.5221 & 1.5922 & 1.5922 & 0.1911 & 0.1911 & 0.0 & 0.0 \\
\hline 0.15 & 2.4198 & 1.3941 & 1.3941 & 0.2091 & 0.2091 & 0.0 & 0.0 \\
\hline 0.17 & 2.3590 & 1.2834 & 1.2835 & 0.2182 & 0.2182 & 0.0001 & 0.0 \\
\hline 0.20 & 2.2770 & 1.1426 & 1.1426 & 0.2285 & 0.2285 & 0.0 & 0.0 \\
\hline 0.22 & 2.2278 & 1.0624 & 1.0624 & 0.2337 & 0.2337 & 0.0 & 0.0 \\
\hline 0.27 & 2.1200 & 0.8983 & 0.8981 & 0.2425 & 0.2425 & 0.0002 & 0.0 \\
\hline 0.30 & 2.0641 & 0.8192 & 0.8190 & 0.2457 & 0.2457 & 0.0002 & 0.0 \\
\hline 0.32 & 2.0299 & 0.7727 & 0.7728 & 0.2473 & 0.2473 & -0.0001 & 0.0 \\
\hline 0.35 & 1.9827 & 0.7109 & 0.7109 & 0.2488 & 0.2488 & 0.0 & 0.0 \\
\hline 0.37 & 1.9536 & 0.6742 & 0.6742 & 0.2495 & 0.2495 & 0.0 & 0.0 \\
\hline 0.40 & 1.9132 & 0.6249 & 0.6250 & 0.2500 & 0.2500 & 0.0001 & 0.0 \\
\hline 0.42 & 1.8882 & 0.5954 & 0.5954 & 0.2501 & 0.2501 & 0.0 & 0.0 \\
\hline 0.45 & 1.8531 & 0.5553 & 0.5554 & 0.2499 & 0.2499 & 0.0001 & 0.0 \\
\hline 0.50 & 1.8008 & 0.4981 & 0.4981 & 0.2491 & 0.2491 & 0.0 & 0.0 \\
\hline
\end{tabular}


TABLE 6

COMPARISONS BETWEEN ANALYTICAL AND NUMERICAL SOLUTIONS OF THE RELATIVISTIC FUNCTIONS (1) AND $\tilde{M}(\sigma)$ FOR $n=1.5$

\begin{tabular}{|c|c|c|c|c|c|c|}
\hline$\sigma$ & $\xi_{1}$ & $\nu\left(\xi_{1}\right)_{N u m}$ & $\nu\left(\xi_{1}\right)_{A n}$ & $\tilde{M}(\sigma)_{N u m}$ & $\tilde{M}(\sigma)_{A n}$ & $\Delta \tilde{M}(\sigma)_{A n}$ \\
\hline 0.0 & 3.6537 & 2.7141 & 2.7141 & 0.0 & 0.0 & 0.0 \\
\hline 0.10 & 3.0384 & 1.4823 & 1.4822 & 0.263592 & 0.263569 & $2.3 \mathrm{E}-05$ \\
\hline 0.12 & 2.9552 & 1.3446 & 1.3446 & 0.274153 & 0.274151 & $2 \mathrm{E}-06$ \\
\hline 0.15 & 2.8464 & 1.1744 & 1.1741 & 0.283069 & 0.282987 & $8.2 \mathrm{E}-05$ \\
\hline 0.17 & 2.783 & 1.08 & 1.08 & 0.285922 & 0.285841 & $8.1 \mathrm{E}-05$ \\
\hline 0.20 & 2.6993 & 0.9602 & 0.9602 & 0.287166 & 0.287166 & 0.0 \\
\hline 0.22 & 2.65 & 0.8925 & 0.8921 & 0.286706 & 0.286558 & 0.000148 \\
\hline 0.27 & 2.5453 & 0.7545 & 0.7543 & 0.282603 & 0.282524 & $7.9 \mathrm{E}-05$ \\
\hline 0.30 & 2.493 & 0.6881 & 0.6881 & 0.278913 & 0.278913 & 0.0 \\
\hline 0.32 & 2.4619 & 0.6496 & 0.6494 & 0.27638 & 0.276292 & $8.8 \mathrm{E}-05$ \\
\hline 0.35 & 2.42 & 0.5982 & 0.5981 & 0.272214 & 0.272151 & $6.3 \mathrm{E}-05$ \\
\hline 0.37 & 2.3949 & 0.5678 & 0.5677 & 0.269366 & 0.269303 & $6.3 \mathrm{E}-05$ \\
\hline 0.40 & 2.361 & 0.5269 & 0.5269 & 0.265018 & 0.265018 & 0.0 \\
\hline 0.42 & 2.3407 & 0.5026 & 0.5025 & 0.262221 & 0.262144 & $7.7 \mathrm{E}-05$ \\
\hline 0.45 & 2.3134 & 0.4696 & 0.4695 & 0.258014 & 0.25794 & $7.4 \mathrm{E}-05$ \\
\hline 0.50 & 2.2749 & 0.4227 & 0.4227 & 0.251326 & 0.251326 & 0.0 \\
\hline 0.52 & 2.2617 & 0.4061 & 0.4061 & 0.248669 & 0.248655 & $1.4 \mathrm{E}-05$ \\
\hline 0.55 & 2.2439 & 0.3835 & 0.3833 & 0.244906 & 0.244817 & $8.9 \mathrm{E}-05$ \\
\hline 0.57 & 2.2333 & 0.3696 & 0.3696 & 0.242484 & 0.242432 & $5.2 \mathrm{E}-05$ \\
\hline 0.60 & 2.2192 & 0.3504 & 0.3504 & 0.238846 & 0.238846 & 0.0 \\
\hline
\end{tabular}

TABLE 7

COMPARISONS BETWEEN ANALYTICAL AND NUMERICAL SOLUTIONS OF THE RELATIVISTIC FUNCTIONS (1) AND $\tilde{M}(\sigma)$ FOR $n=2.0$

\begin{tabular}{|c|c|c|c|c|c|c|}
\hline$\sigma$ & $\xi_{1}$ & $\nu\left(\xi_{1}\right)_{N u m}$ & $\nu\left(\xi_{1}\right)_{A n}$ & $\tilde{M}(\sigma)_{N u m}$ & $\tilde{M}(\sigma)_{A n}$ & $\Delta \tilde{M}(\sigma)_{A n}$ \\
\hline 0.0 & 4.3531 & 2.411 & 2.411 & 0.0 & 0.0 & 0.0 \\
\hline 0.05 & 3.9617 & 1.7165 & 1.7162 & 0.3838 & 0.3838 & 0.0 \\
\hline 0.07 & 3.8443 & 1.5258 & 1.5258 & 0.4037 & 0.4037 & 0.0 \\
\hline 0.10 & 3.6989 & 1.2987 & 1.2983 & 0.4107 & 0.4106 & 0.0001 \\
\hline 0.12 & 3.6191 & 1.1769 & 1.1766 & 0.4077 & 0.4076 & 0.0001 \\
\hline 0.15 & 3.5198 & 1.0272 & 1.0274 & 0.3978 & 0.3979 & -0.0001 \\
\hline 0.17 & 3.4653 & 0.9445 & 0.9440 & 0.3894 & 0.3892 & 0.0002 \\
\hline 0.22 & 3.3619 & 0.7814 & 0.7815 & 0.3665 & 0.3665 & 0.0 \\
\hline 0.25 & 3.3186 & 0.7058 & 0.7056 & 0.3529 & 0.3528 & 0.0001 \\
\hline 0.27 & 3.2962 & 0.6623 & 0.6622 & 0.3441 & 0.3441 & 0.0 \\
\hline 0.30 & 3.2711 & 0.6055 & 0.6057 & 0.3316 & 0.3318 & -0.0002 \\
\hline 0.32 & 3.2595 & 0.5723 & 0.5725 & 0.3238 & 0.3239 & -0.0001 \\
\hline 0.35 & 3.2491 & 0.5285 & 0.5285 & 0.3127 & 0.3127 & 0.0 \\
\hline 0.37 & 3.2463 & 0.5026 & 0.5024 & 0.3057 & 0.3056 & 0.0001 \\
\hline 0.40 & 3.2473 & 0.4680 & 0.4678 & 0.2960 & 0.2959 & 0.0001 \\
\hline 0.45 & 3.2644 & 0.4195 & 0.4196 & 0.2814 & 0.2815 & -0.0001 \\
\hline 0.47 & 3.2754 & 0.4028 & 0.4030 & 0.2761 & 0.2763 & -0.0002 \\
\hline 0.50 & 3.2967 & 0.3800 & 0.3804 & 0.2687 & 0.2690 & -0.0003 \\
\hline 0.52 & 3.3128 & 0.3662 & 0.3668 & 0.2641 & 0.2645 & -0.0004 \\
\hline 0.55 & 3.3416 & 0.3474 & 0.3468 & 0.2576 & 0.2572 & 0.0004 \\
\hline 0.57 & 3.3632 & 0.3359 & 0.3360 & 0.2536 & 0.2537 & -0.0001 \\
\hline 0.60 & 3.3986 & 0.3201 & 0.3202 & 0.2479 & 0.2481 & -0.0002 \\
\hline 0.62 & 3.4253 & 0.3104 & 0.3103 & 0.2444 & 0.2443 & 0.0001 \\
\hline 0.65 & 3.4678 & 0.2970 & 0.2973 & 0.2394 & 0.2397 & -0.0003 \\
\hline 0.67 & 3.4982 & 0.2887 & 0.2891 & 0.2363 & 0.2366 & -0.0003 \\
\hline
\end{tabular}


TABLE 8

COMPARISONS BETWEEN ANALYTICAL AND NUMERICAL SOLUTIONS OF THE RELATIVISTIC FUNCTIONS (1) AND $\tilde{M}(\sigma)$ FOR $n=2.5$

\begin{tabular}{|c|c|c|c|c|c|c|}
\hline$\sigma$ & $\xi_{1}$ & $\nu\left(\xi_{1}\right)_{N u m}$ & $\nu\left(\xi_{1}\right)_{A n}$ & $\tilde{M}(\sigma)_{N u m}$ & $\tilde{M}(\sigma)_{A n}$ & $\Delta \tilde{M}(\sigma)_{A n}$ \\
\hline 0.0 & 5.3552 & 2.1872 & 2.1872 & 0.0 & 0.0 & 0.0 \\
\hline 0.01 & 5.2623 & 2.0281 & 2.0281 & 0.641341 & 0.641341 & 0.0 \\
\hline 0.02 & 5.1793 & 1.88702 & 1.88702 & 0.709633 & 0.709632 & 0.0 \\
\hline 0.03 & 5.1052 & 1.76134 & 1.76131 & 0.733034 & 0.7033021 & $1.3 \mathrm{E}-05$ \\
\hline 0.04 & 5.0393 & 1.648899 & 1.648930 & 0.737410 & 0.737424 & $-1.4 \mathrm{E}-05$ \\
\hline 0.05 & 4.9809 & 1.5479 & 1.548019 & 0.7320 & 0.732013 & 0.0 \\
\hline 0.07 & 4.8841 & 1.3744 & 1.374359 & 0.7069 & 0.706927 & 0.0 \\
\hline 0.12 & 4.7383 & 1.0599 & 1.059908 & 0.6238 & 0.623826 & 0.0 \\
\hline 0.15 & 4.7044 & 0.9261 & 0.925878 & 0.5764 & 0.576204 & 0.0002 \\
\hline 0.17 & 4.7006 & 0.8527 & 0.852617 & 0.5475 & 0.547477 & 0.0 \\
\hline 0.20 & 4.7206 & 0.7606 & 0.760086 & 0.5086 & 0.508299 & 0.0003 \\
\hline 0.22 & 4.7498 & 0.7088 & 0.708902 & 0.4855 & 0.485503 & 0.0 \\
\hline 0.25 & 4.8163 & 0.6426 & 0.642273 & 0.4544 & 0.454155 & 0.0002 \\
\hline 0.27 & 4.8753 & 0.6048 & 0.605615 & 0.4359 & 0.436554 & -0.0007 \\
\hline 0.30 & 4.9855 & 0.5556 & 0.554771 & 0.4112 & 0.410576 & 0.0006 \\
\hline 0.35 & 5.2273 & 0.4896 & 0.490730 & 0.3766 & 0.377450 & -0.0009 \\
\hline 0.37 & 5.3450 & 0.4677 & 0.466891 & 0.3648 & 0.364138 & 0.0007 \\
\hline 0.40 & 5.5448 & 0.438571 & 0.444662 & 0.348782 & 0.348982 & -0.0002 \\
\hline 0.42 & 5.6943 & 0.4214 & 0.421721 & 0.3392 & 0.339499 & -0.0003 \\
\hline 0.45 & 5.9440 & 0.3984 & 0.397734 & 0.3263 & 0.325758 & 0.0005 \\
\hline 0.47 & 6.1284 & 0.3847 & 0.384457 & 0.3186 & 0.318326 & 0.0003 \\
\hline 0.50 & 6.4335 & 0.3664 & 0.366915 & 0.3081 & 0.308537 & -0.0004 \\
\hline 0.52 & 6.6569 & 0.3555 & 0.355406 & 0.3019 & 0.301804 & 0.0001 \\
\hline 0.55 & 7.0239 & 0.3408 & 0.340363 & 0.2935 & 0.293112 & 0.0004 \\
\hline 0.57 & 7.2910 & 0.3320 & 0.331352 & 0.2885 & 0.287911 & 0.0006 \\
\hline 0.60 & 7.7273 & 0.3202 & 0.319843 & 0.2818 & 0.281497 & 0.0003 \\
\hline 0.62 & 8.0423 & 0.3131 & 0.319070 & 0.277851 & 0.277888 & $-3.7 \mathrm{E}-05$ \\
\hline 0.65 & 8.5563 & 0.3036 & 0.305345 & 0.2726 & 0.274169 & -0.0016 \\
\hline 0.67 & 8.9257 & 0.2980 & 0.297274 & 0.2696 & 0.268953 & -0.0006 \\
\hline 0.70 & 9.5224 & 0.2905 & 0.291442 & 0.2657 & 0.266579 & -0.0009 \\
\hline 0.72 & 9.9494 & 0.2860 & 0.284817 & 0.2634 & 0.2623610 & 0.001 \\
\hline
\end{tabular}


TABLE 9

COMPARISONS BETWEEN ANALYTICAL AND NUMERICAL SOLUTIONS OF THE RELATIVISTIC FUNCTIONS (1) AND $\tilde{M}(\sigma)$ FOR $n=3.0$.

\begin{tabular}{|c|c|c|c|c|c|c|}
\hline$\sigma$ & $\xi_{1}$ & $\nu\left(\xi_{1}\right)_{N u m}$ & $\nu\left(\xi_{1}\right)_{A n}$ & $\tilde{M}(\sigma)_{N u m}$ & $\tilde{M}(\sigma)_{A n}$ & $\Delta \tilde{M}(\sigma)_{A n}$ \\
\hline 0.0 & 6.8968 & 2.01824 & 2.01824 & 2.01824 & 2.01824 & 0.0 \\
\hline 0.05 & 6.7074 & 1.42463 & 1.42463 & 1.42463 & 1.42463 & 0.0 \\
\hline 0.07 & 6.7206 & 1.26543 & 1.26542 & 1.26543 & 1.26542 & $1.0 \mathrm{E}-05$ \\
\hline 0.10 & 6.8258 & 1.07845 & 1.07837 & 1.07845 & 1.07837 & $8.0 \mathrm{E}-05$ \\
\hline 0.12 & 6.9521 & 0.979601 & 0.979949 & 0.979601 & 0.979949 & -0.0003 \\
\hline 0.15 & 7.2285 & 0.85958 & 0.859491 & 0.85958 & 0.859491 & $9.0 \mathrm{E}-05$ \\
\hline 0.17 & 7.4751 & 0.794229 & 0.793908 & 0.794229 & 0.793908 & 0.0003 \\
\hline 0.22 & 8.3481 & 0.667954 & 0.667963 & 0.667954 & 0.667963 & $-1.0 \mathrm{E}-05$ \\
\hline 0.25 & 9.0894 & 0.611096 & 0.612004 & 0.611096 & 0.612004 & -0.0009 \\
\hline 0.27 & 9.6994 & 0.579159 & 0.578934 & 0.579159 & 0.578934 & 0.0002 \\
\hline 0.30 & 10.8327 & 0.538631 & 0.540522 & 0.538631 & 0.540522 & -0.0018 \\
\hline 0.32 & 11.7690 & 0.515833 & 0.519000 & 0.515833 & 0.519000 & -0.003 \\
\hline 0.35 & 13.5271 & 0.487068 & 0.488563 & 0.487068 & 0.488563 & -0.001 \\
\hline 0.37 & 15.0007 & 0.471124 & 0.470841 & 0.471124 & 0.470841 & 0.0003 \\
\hline 0.40 & 17.8197 & 0.451585 & 0.452268 & 0.451585 & 0.452268 & -0.0007 \\
\hline 0.45 & 24.9438 & 0.429831 & 0.429350 & 0.429831 & 0.429350 & 0.0005 \\
\hline 0.47 & 29.0538 & 0.424822 & 0.422867 & 0.424822 & 0.422867 & 0.0020 \\
\hline 0.50 & 37.2058 & 0.421395 & 0.421807 & 0.421395 & 0.421807 & -0.0004 \\
\hline 0.53 & 48.5317 & 0.423168 & 0.418075 & 0.423168 & 0.418075 & 0.0051 \\
\hline 0.60 & 91.0723 & 0.449319 & 0.453089 & 0.449319 & 0.453089 & -0.004 \\
\hline 0.70 & 162.5832 & 0.526621 & 0.529641 & 0.526621 & 0.529641 & -0.003 \\
\hline 0.74 & 177.9357 & 0.558153 & 0.554724 & 0.558153 & 0.554724 & 0.003 \\
\hline 0.75 & 180.4379 & 0.565394 & 0.541169 & 0.565394 & 0.541169 & 0.0242 \\
\hline
\end{tabular}

TABLE 10

THE CRITICAL VALUES $\sigma_{C R}$ CORRESPONDING $\tilde{M}(\sigma)$ FOR VARIOUS INDICES $N$

\begin{tabular}{cccc}
\hline$n$ & $\xi_{1}$ & $\sigma_{\text {critical }}$ & $\tilde{M}(\sigma)$ \\
\hline 1.0 & 1.8882 & 0.42 & 0.20 \\
1.5 & 2.6993 & 0.10 & 0.287166 \\
2.0 & 3.6989 & 0.04 & 0.410546 \\
2.5 & 5.0393 & 0.0 & 0.737424 \\
3.0 & 6.8968 & 0.53 & 2.01824 \\
3.0 & 48.5317 & & 0.416203 \\
\hline
\end{tabular}




\section{APPENDIX B. THE SERIES ACCELERATION} TECHNIQUE

To accelerate the convergence of the series solution of equation 13, we followed the scheme developed by Nouh (2004). As the first step of this scheme, the alternating series is accelerated by Euler-Abel transformation (Demodovich \& Maron 1973). Let us write

$$
\theta(\xi)=a_{0}+\xi \phi(\xi)
$$

where

$$
\phi(\xi)=\sum_{k=0}^{\infty} a_{k} \xi^{k-1}=\sum_{k=1}^{\infty} a_{k+1} \xi^{k}
$$

then

$$
(1-\xi) \phi(\xi)=\sum_{k=0}^{\infty} a_{k+1} \xi^{k}-\sum_{k=1}^{\infty} a_{k} \xi^{k}=a_{0}+\sum_{k=0}^{\infty} \Delta a_{k} \xi^{k},
$$

where $\Delta a_{k}=a_{k+1}-a_{k}, k=0,1,2, \ldots$ are finite differences of the first order of the coefficients $a_{k}$. Applying the Euler-Abel transformation to the power series $\sum_{k=0}^{\infty} \Delta a_{k} \xi^{k}, \boldsymbol{p}$ times, and after some manipulations we obtain

$$
\sum_{k=0}^{\infty} a_{k} \xi^{k}=\sum_{i=0}^{\infty} \Delta^{i} a_{0} \frac{\xi^{i}}{(1-\xi)^{i+1}}+\left(\frac{\xi}{1-\xi}\right)^{p} \sum_{k=0}^{\infty} \Delta^{p} a_{k} \xi^{k},
$$

where $\Delta^{0} a_{0}=a_{0}$. Equation (25) becomes meaningless when $\xi=1$, so, by setting $\xi=-t$, we obtain the Euler-Abel transformed series as

$$
\begin{aligned}
\theta E_{n}(t)= & \sum_{k=0}^{\infty} \Delta^{i} a_{0} \frac{t^{i}}{(1-t)^{i+1}}+ \\
& \left(\frac{t}{1-t}\right)^{p} \sum_{k=0}^{\infty} \Delta^{p}\left[(-1)^{k} a_{k}\right] t^{k} .
\end{aligned}
$$

Returning to the earlier variable, $\xi$, we obtain

$$
\begin{aligned}
\theta E_{n}(\xi)= & \sum_{i=0}^{p-1}(-1)^{i} \Delta^{i} a_{0} \frac{\xi_{i}}{(1+\xi)^{i}}+ \\
& \left(\frac{\xi}{1+\xi}\right)^{p} \sum_{k=0}^{\infty}(-1)^{k+p}\left[\Delta^{p} a_{k}\right] \xi_{k},
\end{aligned}
$$

where

$$
\Delta^{p} a_{k}=\Delta^{p-1} a_{k+1}-\Delta^{p-1} a_{k} .
$$

Any order difference $\Delta^{p} a_{k}$ can be written as a linear combination

$$
\Delta^{p} a_{k}=\sum_{i=0}^{p}(-1)^{p-i}\left(\begin{array}{c}
p \\
i
\end{array}\right) a_{k+1},
$$

where

$$
\left(\begin{array}{c}
p \\
i
\end{array}\right)=\frac{p !}{i !(p-i) !} .
$$

The second step is to apply Padé approximation to the Euler-Abel transformed series, equation 27. 


\section{REFERENCES}

Araujo, F. M. \& Chirenti, C. B. M. H. 2011, arXiv:1102.2393

Bardeen, J. M., Thorne, K. S., \& Meltzer, D. W. 1966, ApJ, 145, 505

Bludman, S. A. 1973, ApJ, 183, 637

Bonnor, W. B. 1958, MNRAS, 118, 523

Casalbuoni, R. \& Nardulli, G. 2004, RvMP, 76, 263

Chandrasekhar, S. 1964, ApJ, 140, 417

Chu, P. Ch., Wang, X., Chen, L. W., \& Huang, M. 2015, PhRvD, 91, 3003

Demidovich, B. P. \& Maron, I. A. 1973, Computational Mathematics, 519.4, D4

Ferrari, L., Estrela, G., \& Malheiro, M. 2007, IJMPE, 16, 2834

Horedt, G. P. 2004, Polytropes-Applications in Astrophysics and Related Fields, Astrophysics and Space Science Library 306 (Dordrecht: Springer)

Horedt, G. P. 2013, ApJ, 773, 131

Isayev, A. A. 2015, PhRvC, 91, 5208

Khalilov, V. R. 2002, PhRvD, 65, 6001
Landau, L. D. \& Lifshitz, E. M. 1975, The classical theory of fields (2nd ed; Reading, MA: Addison-Wesley Publishing Co.)

Nouh, M. I. 2004, NewA, 9, 467

Nouh, M. I. \& Saad, A. S. 2013, International Review of Physics, 7(1), 16

Pascual, P. 1977, A\&A, 60, 161

Raga, A. C., Osorio-Caballero, J. A., Chan, R. S., et al. 2020, RMxAA, 56, 55

Saad, A. S. 2004, AN, 325, 733

Sen, N. R. \& Roy, T. C. 1954, ZA, 34, 84

Shapiro, S. L. \& Teukolsky, S. A. 1983, Black-holes, white dwarfs and neutron stars: the physics of compact objects (New York, NY: Wiley)

Sharma, J. P. 1988, ApJ, 329, 232

Takatsuka, T. \& Tamagaki, R. 1993, PthPS, 112, 27

Tolman, R. C. 1939, PhRv, 55, 364

Tooper, R. F. 1964, ApJ, 140, 434

Zeldovich, Y. B. \& Novikov, I. D. 1978, Relativistic Astrophysics, Vol. I: Stars and relativity (Chicago, IL: University of Chicago Press)

Tarek M. Kamel, Mohamed I. Nouh, Abdel-Naby S. Saad, and Ashraf A. Shaker: Astronomy Department, National Research Institute of Astronomy and Geophysics(NRIAG), 11421 Helwan, Cairo, Egypt.

Abdel-Naby S. Saad: Department of Mathematics, Deanship of Educational Services, Qassim University, Buraidah, Saudi Arabia (saad6511@gmail.com). 